\title{
ASORS beim DKK 2016: Highlights zur Supportivtherapie
}

Der Deutsche Krebskongress (DKK) 2016 stand unter dem Motto „präventiv, personalisiert, präzise und partizipativ“. Das sind genau die Stichworte, die auch auf die Supportivtherapie zutreffen. Die ASORS konnte sich als Arbeitsgemeinschaft der Deutschen Krebsgesellschaft (DKG) mit 25 wissenschaftlichen Symposien, die sie gemeinsam mit anderen Arbeitsgemeinschaften der DKG auf dem Krebskongress gestaltete, sehr präsent positionieren. Dies zeigt deutlich, wie wichtig die Supportivtherapie im Rahmen der onkologischen Behandlungskonzepte ist.

\section{Querschnittsdisziplin}

Was auf dem DKK auch sehr klar zum Tragen kam, war, dass die Supportivtherapie eine Querschnittsdisziplin ist. Sie vereint zum einen viele verschiedene medizinische Fachrichtungen, aber sie vernetzt uns Mediziner auch mit anderen Berufsdisziplinen wie beispielsweise Psychologie, Pflege und Pharmazie. In der ASORS ebenso wie in unserer internationalen Fachgesellschaft, der MASCC (Multinational Association of Supportive Care in Cancer) leben wir das Konzept der Interdisziplinarität und der Multiprofessionalität von Anfang an. Für mich persönlich war faszinierend, wie diese Zusammenarbeit auch im Hinblick auf die Gestaltung der DKK-Symposien geklappt hat.

Supportivtherapie und Palliativmedizin

Ein großes Highlight war für mich die Plenarsitzung zum Thema "Early integration of palliative and supportive care" mit der Keynote von Gary Rodin aus Toronto. Die

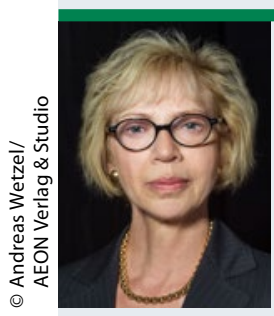

„Die Supportivtherapie ist die Basis jeder onkologischen Behandlung."

Prof. Dr. med. Petra Feyer, Berlin

Vorsitzende des Bereichs Supportivtherapie der Arbeitsgemeinschaft Supportive Maßnahmen in der Onkologie, Rehabilitation und Sozialmedizin (ASORS) der Deutschen Krebsgesellschaft.
Beiträge der Referenten haben sehr deutlich gezeigt, wie wichtig eine frühzeitige Integration der Palliative Care bei fortgeschrittenen Tumoren ist. Die rege Diskussion mit teilweise sehr unterschiedlichen Positionierungen hat deutlich gemacht, dass wir durchaus noch Klärungs- und Definitionsbedarf bezüglich palliativer und supportiver Maßnahmen haben.

\section{S3-Leitlinie Supportive Therapie}

Sehr wichtig für uns als ASORS war natürlich auch die Vorstellung des Arbeitsstands unserer S3-Leitlinie "Supportive Therapie bei onkologischen Patientlnnen", die von Frau Prof. Jordan aus Halle koordiniert wird. Diese Querschnittsleitlinie mit zehn wichtigen Themen zur onkologischen Supportivtherapie wird gemeinsam mit der DGHO (Deutsche Gesellschaft für Hämatologie und Medizinische Onkologie) und der DEGRO (Deutsche Gesellschaft für Radioonkologie) erstellt. Die Leitlinienarbeit ist im Wesentlichen abgeschlossen. In zwei Konsensuskonferenzen im Jahr 2015 wurden die Schlüsselempfehlungen bestätigt und wir gehen davon aus, dass wir im Mai 2016 die Vollpublikation dieser wichtigen S3-Leitlinie vorliegen haben. In zwei Sitzungen beim DKK wurde thematisiert, wie wichtig es ist, diese Leitlinien nicht nur zu erstellen, sondern auch dafür zu sorgen, dass sie in der Onkologie breit angewendet werden. Es war uns wichtig, zu kommunizieren, dass eine leitliniengerechte Supportivtherapie kostengünstig, effizient und patientengerecht ist - und ich glaube, es ist uns gelungen, diese Botschaft zu vermitteln.

\section{Bewegung und Ernährung}

Ganz relevante Themen, die früher von Onkologen als supportive "Nebenschauplätze" betrachtet wurden, sind heute Bereiche, die für die Lebensqualität der Patienten besonders wichtig sind. Das sind Bereiche, auf die sie selbst auch Einfluss nehmen können. Damit spreche ich z. B. den Komplex „Bewegung und körperliche Aktivität" an. Es gibt inzwischen zahlreiche wissenschaftliche Daten, die zeigen, dass Bewegungsprogramme einen positiven Einfluss auf das (Über)leben haben. Die Ernährung spielt in allen onkologischen Konzepten eine Rolle, sowohl in der Prävention als auch während und nach einer Tumortherapie. Dazu gab es beim DKK zahlreiche Beiträge mit hochrangigen Referenten, beispielsweise das Symposium zur Ernährung „Kachexie, Sarkopenie und Krebs" unter Federführung von Herrn Prof. Plauth, der gleichzeitig der Vorsitzende der Deutschen Gesellschaft für Ernährungsmedizin (DGEM) ist, sowie Herrn Dr. Jann Arends, der innerhalb der ASORS die Expertengruppe Ernährung leitet. Zur klinischen Ernährung in der Onkologie wurden im letzten Jahr Leitlinien der DGEM veröffentlicht.

\section{Organbezogene Kasuistiken}

Dann haben wir sehr interessante Falldiskussionen durchführen können, bei denen unterschiedliche Supportivmaßnahmen organbezogen beleuchtet wurden. Zum Beispiel: Antiemese beim Mammakarzinom - was ist zu beachten? Es sind aktuell die neuen MASCC-Leitlinien zur Antiemese verabschiedet worden. Hier haben sich Änderungen im Vergleich zur Vorversion ergeben und diese konnten praktisch druckfrisch an das Auditorium weitergegeben werden.

\section{Komplementäre Maßnahmen}

Ein wichtiges Thema ist weiterhin die Nutzung komplementärmedizinischer Maßnahmen. Auch hier denke ich, müssen wir weitere Studien durchführen, die zeigen, welche komplementärmedizinischen Maßnahmen unseren Patienten nutzen. Dabei ist das Gespräch mit dem Arzt äußerst wichtig, um Interaktionen mit den tumorspezifischen Therapien zu vermeiden.

\section{Management zielgerichteter Therapien}

Ein weiteres Highlight war meiner Ansicht nach die Beleuchtung des erforderlichen multiprofessionellen Managements von Nebenwirkungen zielgerichteter Therapien. Auch die neuen Immuntherapien wie z.B. die CTLA4-Inhibitoren und die PD-1-/PD-L1-Inhibitoren bringen $\mathrm{Ne}$ benwirkungen mit sich, mit denen wir erst lernen müssen umzugehen. Spektrum und Zeitpunkt des Auftretens sind anders. Die Langzeitkomplikationen kennen wir noch gar nicht. Ziel ist es auch hier, präventiv zu denken: also Prädiktoren zu entwickeln, die uns sagen, der Patient wird mit großer Wahrscheinlichkeit diese oder jene Nebenwirkung entwickeln. Nur so können wir uns sowohl über Prophylaxe als auch Therapie Gedanken machen. Dazu gibt es noch sehr viel Forschungsbedarf. 